\title{
The Design and implementation of network based on SDN Technology
}

\author{
Jinyi Zhou \\ Sichuan Information Technical College, China \\ 30940350@qq.com
}

Keywords: SDN technology; Network; The network architecture; Network management

\begin{abstract}
In this paper, we propose a SDN based on the information network architecture to solve the problems existing in the current network, and try to avoid the problems existing in the information network. First the research development on the SDN technology, the information network were detailed introduction and analysis, through the analysis of the Internet's development and current problems existed in, then illustrates the network information becomes the trend of the future development of the network, and use SDN to solve the problems in network information bring benefits.
\end{abstract}

\section{Introduction}

In the current Internet, more and more applications in the network, leading to a surge in traffic network. In May 2012, Cisco on global IP traffic prediction by 2016, global IP traffic will reach 1.3zettabytes (i.e. 13 million TB), 2011 to 2016, an increase of 4 times and Wi Fi will be accounted for half of the DP Flow [1]. More and more information transmitted in the network. But in the current network, the transmission of information is completely based on the address information. The information content is transparent to the network. So a lot of the same information transmission network, the bandwidth and energy waste [2]. Also from the perspective of social economics, the current network architecture for information service providers, profit is relatively low. So I hope future network can provide a new publication and subscription content, different people can provide different services, in order to protect the stakeholders of the benefits of [3]. We need new network architecture to distribute information effectively.

\section{SDN technology and information network}

\subsection{SDN Technology Overview}

SDN (Software Defined Network) [4] is a recently emerging network technology. It makes the network structure of innovation, while allowing network operators more control. It customize and optimize their own infrastructure, it is possible to reduce the overall capital and operating costs. SDN technology ideas: SDN is new network architecture, the network control and forwarding separation, and can be directly programmed. Will control the migration to the computing device (formerly separate network equipment and tightly bound together), upper layer applications and services for infrastructure abstraction, the network as a logical or virtual entity.

\subsection{Information network}

The research of network information in full swing, and different working group are put forward for different network architectures, has yet to form a unified standard [5]. In the present study, the naming of the information in the network consensus has been reached, each item of information named in different forms, but need information in the network can be uniquely identifies. Some information network is the design based on routing information name, such as NDN; some information network design using information name retrieval, but transmission of information not use name information routing, such as PSIRP, NDN.

\subsection{Remaining issues in the information network}

There are many research organizations in the information network, in different research programs, there are some problems, summarized as follows: 
1) The distribution of information is not controllable. In the research of network information, in the information distribution, considering only the information to reach the request, but the transmission of information, without considering the controllability of the information, that is, the network can control information transmission. If the wrong information in the network transmission path selection and control, the transmission of information may cause the transmission link and can't meet the transmission requirements of the information itself, so the network did not complete information, transmission.

2) The information routing process to achieve complex. The path calculation in PSIRP based on Bloom filter calculation routing. Routing calculation errors caused by this way, namely the information reaches the end user, the user may do not need this information, resulting in bandwidth waste. Routing information based on the name, resulting in the routing table to store information name [6]. Due to the name of information is the only and the amount of information will be very large, will lead to the routing table of large scale, so as to make the information retrieval will be more complex. In the design of some of the information network, using DHT for name resolution, due to the size of the network is very large, when using the DHT, affecting hit rate and implementing complex.

3) In the current network deployment complex. Due to the large number of applications already exist now in the network, and the network for human life is an essential part of. Study on the existing information network for subversive network architecture, the theory is feasible, but large-scale deployment is not realistic, the cost will be very large, and in the network transmission is not the result of practical considerations. But like NDN can as the overlay network, but in the deployment of all aspects related to some problems, such as router cache is not big enough and so on. Therefore, to achieve a truly convenient deployment in the network information network is an urgent problem to be solved.

\subsection{SDN and the benefits of information network combination}

The current SDN attention from all walks of life, a high degree of recognition and commercialization in slowly [7]. It has good feasibility in SDN, it is easy to deploy in the network. And the control plane and data plane separation of the idea of SDN, is in line with the information in the network of information is highly controllable. The use of SDN to achieve the benefits of information network, the specific performance is the following:

1) Easy to control the transmission of information. In the information network, the network needs to transmit control information, select the best path for information transmission. In SDN control plane is separated from the data plane and control plane data plane control strategy is flexible and variable. The transmission control plane of the SDN to control the information is reasonable.

2) Easy to implement and deploy. Some of the products currently have SDN, so the feasibility of the deployment of SDN is no problem. Because SDN is open source code, based on the extended SDN network, more conducive to reducing the development cycle.

3) Easy to be recognized by the public. Because SDN is of many well-known enterprises and service providers were investigated, indicating that it is recognized by the public, therefore using SDN to construct a network of information, and other information networks are compared, are more likely to be accepted by the public.

\section{SDN-ICN network architecture}

\subsection{The overall goal of SDN-ICN}

The main goal of SDN-ICN is to build an efficient and scalable network by using the idea of SDN. To meet the large-scale transmission of information and can be deployed in the network. The specific performance of the following points:

1) To meet the efficient transmission of information. Ensure the efficient transmission of information in the network. 
2) To solve a series of problems existing in the current network in essence. The network design does not consider the mobility management and multicast problem, so for this a series of problem solution efficiency is not high enough.

3) Network can effectively control the transmission of information. According to the state information and network information, can adaptively adjust the transmission path.

4) Network with high efficient scalability. If the expansion of network scale, SDN-ICN network architecture can well support.

\subsection{SDN-ICN features}

1)Using SDN to construct the information network, through the control plane can realize the effective control of the network of information, when changes in the network state, the control plane can be timely adjustment of information transmission, to ensure that the information in the whole process of transmission, are able to transmit.

2) Construction distribution path. In the construction of distribution path, according to the characteristics of information and network status information, to select the most suitable for the transmission path.

3) Support for mobility in nature. Considering to the complexity of network mobility support now, in network design, from the nature of mobility support, without too much information interaction and design a network, making the overhead of the network reduced.

4) Essentially support multicast. Due to the existing network multicast is not considered in the design stage, leading to the multicast support complex. SDN-ICN use network for information dissemination path controllability, which makes the information in transmission, multicast, thereby saving network bandwidth.

\subsection{SDN-ICN architecture design}

Use SDN idea to design information network architecture, the whole network is divided into a control plane and data plane, control plane is responsible for the information distribution path is constructed and cache management, data plane is responsible for transfer of information. The control plane and data plane completely that, between the control plane and data plane using standard communication protocol. At the same time, the control plane network entities of the data plane using different control strategies, making the network more flexible and diversity. Network architecture as shown in figure 3-1:

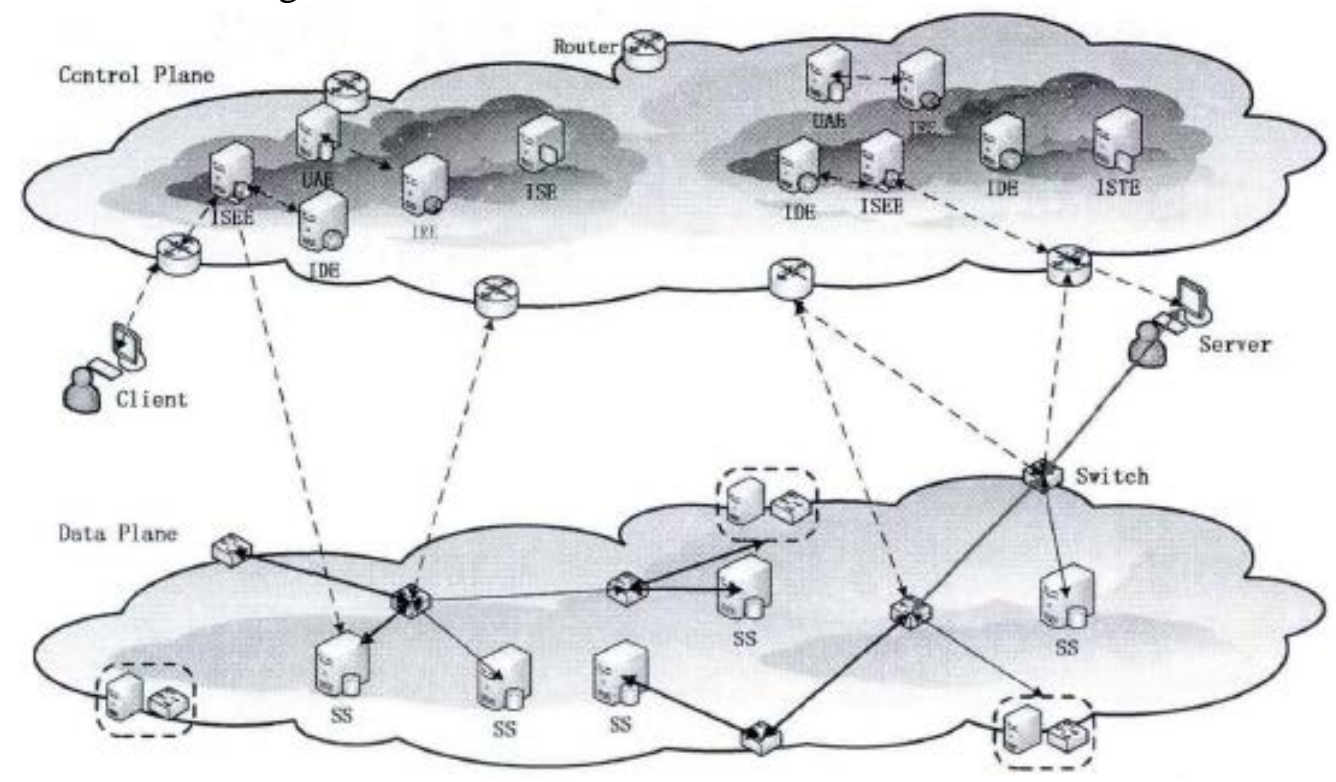

Figure 3-1 SDN-ICN network architecture

\subsection{Functional entity}

UAE (User Authentication Entity): user authentication entity. When the user releases the information, UAE is responsible for the identity of the user authentication. If authentication is successful, UAE can also return to the public / private key for IRE, ID used when generating information. 
IRE (Information Register Entity) [8]: the user will need to register information system to be released. ISE (information Search entity), it contains a series of attribute information, such as ID information, type information, where the information server, cache list. At the same time the ire contains enough information, so ire has a function is information retrieval, namely when a user submits a request, the first to reach the ire of information retrieval.

IDE (Information Delivery Entity): information distribution entity, to construct a path of information distribution. IDE is responsible for the request message from the IRE distribution path structure.

ISTE (Information Store Entity): information storage location. A series of location information, you can query to the nearest user location information. At the same time is also stored in the use of multicast to the.

SS (storage switch): only in the data plane of network entities, accept control message from the IDE, responsible for transfer of information.

Request entity for Client: information. The function of the network entity in the network and the network entity is the same, the service request is Client.

Owner of Server: information. A series of information is stored in the Server [9].

By setting the cache, reducing network bandwidth, improve the response speed. In the information registration, information retrieval, tectonic distribution path, choose the location cache etc. were completed by the control of the plane, the transmission of information in the data plane.

\section{Conclusion}

This paper introduced the development of SDN technology, information network and research status. On the information network of the origin, the research project also carried out a thorough introduction. Analysis the problems that still exist in the existing information network research, and puts forward using SDN technology to design new information network architecture, and describes the use of SDN to network architecture design brought benefits, solved the problems in network information of using SDN.

\section{References}

[1] H.Yin, H.Xie. SDNi: A Message Exchange Protocol for Software Defined Networks (SDNS) across Multiple Domains. IETF draft. 2012.

[2] H.Xie, T.Tsou. Use Cases for ALTO with Software Defined Networks.TF draft. 2012.

[3] J. Medved,A. McLachlan. MPLS-TP PseudoWire Configuration using OpenFlow 1.3. IETF draft. 2012.

[4] Li Erran Li,Morley Mao. Toward Software-Defined Cellular Networks. 2010

[5]Lixia Zhang,D.Estrin, J.Burke. Nmned Data Networking (NDN) Project. PARC Technical Report NDN-0001. Oct. 2010.

[6] B.Ahlgren, M.D'Ambrosio, C.Dannewitz. Second Netlnf Architecture Description. FP7-ICT-2007-1 -216041 -4WARD/D-6.2. Jan. 2010.

[7] Jarschel, M. \& S. Oechsner, D. Schlosser, R. Pries, S. Goll,PhuocTran-Gia. Modeling and Performance Evaluation of an OpenFlow Architecture[C]. ITC, 2011.

[8] Bianco, A. \& R. Birke,L. Giraudo. OpenFlow Switching: Data Plane Performance[C], Cape Town: ICC, 2010.

[9] Azodolmolky,S. \& R, Nejabati,E. Escalona, R. Jayakumar, N. Efsathiou, D. Simeonidou. Integrated OpenFlow-GMPLS Control Plane: An Overlay Model for Software Defined Packet over Optical Networks[C]. Geneva, Switzerland: ECOC, 2011. 